\title{
TWO CASES OF PATENCY OF THE DUCTUS
}

\section{ARTERIOSUS}

\author{
BY T. WARDROP GRIFFITH
}

THE two examples of patency of the ductus arteriosus on which I desire to comment occurred in patients in whom the affection was recognized during life. One of these succumbed to infective endocarditis, and the diagnosis was verified on the post-mortem table. In the other the condition was probably associated with some further anomaly of the great vessels and with a developmental error which had given rise to cyanosis, for this is a symptom which is not often met with in uncomplicated cases of patency of the arterial duct.

\section{CASE I.}

The first patient, a young unmarried woman of 32, very anaemic, somewhat wasted and short of breath, was sent to see me by Dr. Eley of Batley. From her earliest childhood she had been short of breath and she had been unable to join in the more energetic games of her fellows. Her mother volunteered the statement that she had always had a large heart, as she had noticed it beating with undue violence when she was a baby. This had also been noticed by the brothers of the little girl, who, being a few years older, had had the duty imposed upon them of carrying her about.

After examining the patient I wrote to Dr. Eley as follows: 'I think there are really two conditions, a congenital malformation-and here I incline to patency of the ductus arteriosus-and an acquired disease of the mitral valve with both stenosis and regurgitation.'

On March 10, 1903, a few days after I had seen the patient at my rooms, she was, at the request of Dr. Eley, admitted under my care into the General Infirmary at Leeds. The diagnosis of mitral disease was confirmed; a crescendo presystolic bruit at the apex followed by a systolic bruit made the matter clear. Further examination led me to suspect the presence of aortic disease, and as the days passed I became convinced of this. The temperature, which was not quite steady when she was admitted, began to show marked oscillations, and it became increasingly obvious that we had to do with a case of infective endocarditis complicating an old cardiac lesion. There was marked pyorrhoea alveolaris.

The presence of a systolic and diastolic bruit due to aortic disease introduced an element of difficulty into the diagnosis of the condition of patent ductus arteriosus. This was made on the following grounds. At the inner end of the second left intercostal space there was heard a loud systolic bruit, followed by a very loud diastolic bruit which was audible a considerable distance outwards along the second space and was not transmitted along the left edge of the sternum. I was particularly struck by the continuity of the two bruits; the 
only way in which they could be regarded as separated from one another was by the occurrence of the greatly accentuated second sound which was heard with its maximum at the inner end of the second space. As I repeatedly pointed out to my class of students, there were not two bruits, but one continuous bruit with systolic and diastolic increments, and so striking was this that I frequently said the sound was not adequately described as 'to and fro' but rather as ' to and to'. It gave me the impression of a sound caused by the passage of fluid in a continuous stream, in the same direction, but with varying degrees of force. The systolic and diastolic increments were, I thought, most striking at the beginning of these periods ; certainly this was the case in respect of the diastolic increment. No one has described this physical sign better than the late Dr. G. A. Gibson (1), who wrote as follows: "Beginning distinctly after the first sound, it accompanies the latter part of that sound, occupies the short pause, accompanies the second sound, which may be accentuated in the pulmonary area, or may be and often is doubled, and finally dies away during the long pause.'

When this physical sign has been definitely established, a diagnosis of patency of the ductus arteriosus or of some other kind of communication between the aorta and pulmonary artery may safely be made. The alternatives I considered were the following:

1. That the signs were altogether due to disease of the pulmonary segments. Against this was the fact that, though there was marked venous pulsation in the neck, there was no great dilatation of the right side of the heart; the second sound of the heart in the pulmonary area was loud, and there was no cyanosis. The rarity of disease of the pulmonary valves of a kind likely to produce signs resembling those met with perhaps influenced me against this diagnosis, though, as we had reason to believe the patient was the sulject of infective endocarditis, a well-known cause of disease of the pulmonary segments was present. In this connexion I was not ummindful of a case I had put on record in 1906 in which infective endocarditis was confined to the pulmonary valve (2).

2. That the signs were altogether due to disease of the aortic segments. Now there was no doubt that disease of the aortic valve was present, and the signs of regurgitation became increasingly obvious. Direct and regurgitant bruits were heard in the usual situation and the pulse was of the Corrigan character. It is true that the bruits of aortic disease are sometimes heard better to the left of the sternum than to the right, but in this case the excess in loudness at the inner end of the second left space was so great as to demand some other explanation.

These considerations, coupled with the remarkable continuity of the systolic and diastolic parts of the bruit, led me to diagnose patency of the ductus arteriosus.

Very distinct pulsation could be seen and felt towards the inner end of the second and third left interspaces. Dr. Rowden, who is in charge of the radiographic department of the Infirmary, was good enough to make an examination of the patient in my presence with the fluoroscopic screen. This revealed the presence of a pulsating area in the situation of the stem of the pulmonary artery, as though that vessel were greatly dilated.

The assistance which may be obtained by the use of the orthodiagraph in the diagnosis of patency of the ductus arteriosus has been well emphasized by Wessler and Bass (3). With their permission I reproduce one of the diagrams in their paper, in which the dilatation of the pulmonary artery under the unwonted strain of the aortic pressure acting through the patent ductus is well seen (Fig. 1).

3. There remained of course a third alternative diagnosis, namely a communication between the aorta and pulmonary artery due to an aneurism of one or the other. The age and sex of the patient made this very unlikely. In 
connexion with endocarditis of the infective variety it is well known that aneurisms of the stem of the pulmonary artery or of the aorta may arise, and that communications may in this way be caused. In this case, however, there was every reason to think that the infective endocarditis was of recent origin, and that some abnormal condition of the heart associated with enlargement had been present from earliest childhood and probably from birth.

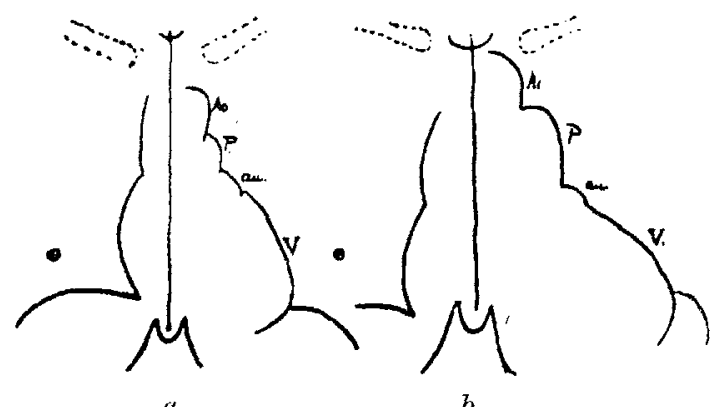

Frg. 1. After Wessler and Bass. (a) Orthodiagram; normal condition; (b) Enlarged (and pulsating) pulmonary artery $(P)$.

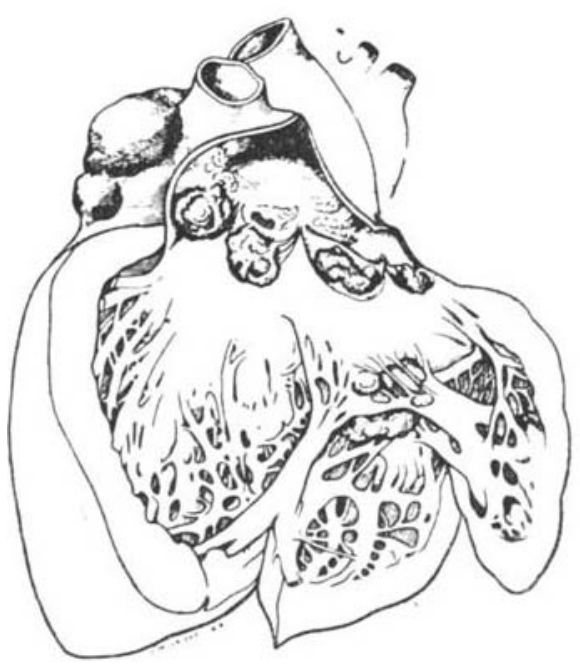

FId. 2. Shows the mitral stenosis; infective mitral and aortic valvulitis and the bilobed aneurysmal dilatation of the pulmonary artery.

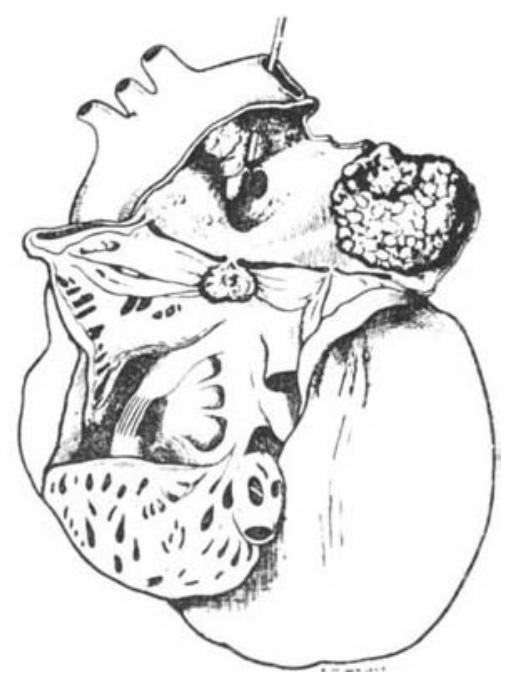

Fra. 3. Shows the mass of vegetations in the pulmonary artery; a probe is passed along the patent ductus arteriosus.

The patient remained under my observation till her death on July $27,1913$. It is not relevant to my subject to comment on the treatment which was followed. At one time the intensity of the infective process seemed to be lessening, but during the last few weeks of her life the patient got steadily weaker and it was clear that the end was imminent. She died rather suddenly after an attack of lirgent dyspnoea.

During the last few weeks of her life we were struck by the complete disappearance of the characteristic continuous bruit which I have described and on which I had relied in the diagnosis of the condition.

At the antopsy, which was made by Dr. Matthew J. Stewart, there was 
found considerable enlargement of the heart, which weighed about $14 \frac{1}{2}$ oz. There was marked hypertrophy without notable dilatation of the right ventricle. There was recent pericarditis, and before the heart was removed there was found on the anterior and left aspect of the pulmonary artery, which was generally dilated, a large bilobed aneurysmal swelling, which is well seen in Fig. 2. There was old-standing disease of the mitral valve with moderate stenosis of the orifice, and both this valve and the aortic segments presented large fungating masses of vegetations. Evidence of recent endocarditis was found at the junction of the two posterior segments of the pulmonary valve (Fig. 3). The lumen of the pulmonary artery was almost occluded by a large mass of blood-stained vegetations, which was gently adherent to the walls of the vessel at two situations. One of these was at the anterior and left aspect of the artery, where there was a double concavity corresponding to the convexity seen from the outside; the other was around the opening of the ductus arteriosus, and into this there projected a conical mass of vegetations so as completely to occlude the aperture. When the ductus was cleared of this it permitted the passage of a No. 9 catheter. The space left for the passage of the blood between the mass of vegetations and the wall of the vessel was probably much greater during life than one might expect from an inspection in the post-mortem room, where the dilating influence of the blood-pressure would be absent.

The diagnosis of patent ductus arteriosus was therefore confirmed and the disappearance of the characteristic sign of the continuous bruit accounted for by the occlusion of the aperture.

It must be remembered that while the function of the ductus arteriosus during foetal life is to convey the greater part of the blood which leaves the right ventricle into the descending part of the aorta, its patency after the establishment of the ordinary circulation will be attended with a flow in the opposite direction, namely from the aorta to the pulmonary artery, and that this will be the case both during the systole and during the diastole of the heart. The pulmonary artery will therefore be subjected to the pressure of the powerful left ventricle and will be liable to undergo dilatation. Then we must remember that those $w$ ho are the subjects of congenital malformations of the heart are peculiarly liable to endocarditis both of the simple and of the malignant variety. In making an analysis of some sixty cases of malignant endocarditis of the pulmonary valve Dr. Newton Pitt (4) found patency of the interventricular septum in ten cases, congenital pulmonary stenosis in eight, and patency of the ductus arteriosus in three. Should this complication occur, and especially, as in this case, should the wall of the artery be profoundly affected, the occurrence of great aneurysmal dilatation may be readily understood.

In this connexion I may refer to a case put on record by the late James Foulis (5) in 1884. From the figure which is reproduced from his paper (Fig. 4) it will be seen that there is aneurysmal dilatation of the left and anterior wall of the pulmonary artery with extensive infective pulmonary valvulitis and endarteritis. Foulis states that on the wall of the aorta opposite to the opening of the ductus into that vessel there was a bulging, one inch by half an inch in extent, partly filled with coagulum. He is clearly of opinion that the systolic part of the bruit which was heard was due to the passage of blood from the pulmonary artery to the aorta, and that the diastolic part was due to blood 


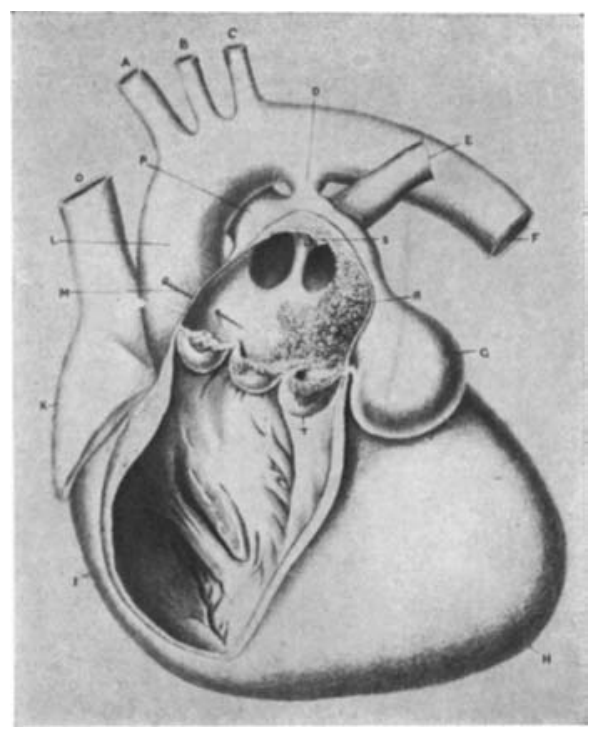

FIG. 4. From the paper by Foulis. 
flowing in the opposite direction. As he points out, the long axis of the patent duetus would, if prolonged in either direction, impinge on the pulmonary artery and aorta at the parts where these vessels are diseased. Yet it is hard to understand how blood can be driven from the pulmonary artery into the aorta, in which one must suppose the blood-pressure to be higher; and it is especially difficult to admit this in the case recorded by Foulis, for it is stated that the right ventricle was thin-walled and that the wall of the left ventricle was thickened. If it is the case that sometimes the right ventricle bursts open the pulmonary valve before the left ventricle has overcome the aortic pressure, we might understand a very transient flow from pulmonary artery to aorta; apart from this it is hard to see how it could occur as the result of simple patency.

Those who have many opportunities of making autopsies in cases of congenital malformations of the heart, especially when the symptom of cyanosis has been present, meet with many examples of patency of the ductus arteriosus. Perhaps the commonest cause of this is the condition usually spoken of as
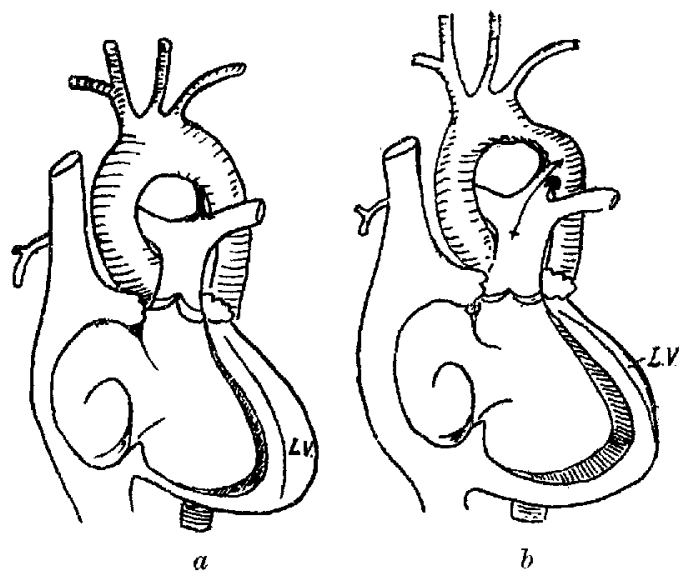

Fra. 5. (a) Normal condition; (b) Coarctation of aorta ; $\mathrm{LY}=$ left ventricle.

congenital 'pulmonary stenosis'. Arthur Keith (6) has shown us that this is indeed seldom due to an inflammatory process affecting the valves, but to an imperfect development of the bulbus cordis. The result of it is that the bloodpressure in the pulmonary artery is so low in consequence of the lessened flow from the right side of the heart that the ductus cannot close, there being a continuous flow from the aorta to the pulmonary artery, and in this way the lungs get their blood supply.

In the interesting condition known as coarctation of the aorta, if there be much constriction of the vessel, the ductus may remain patent, and may be the means of transmitting blood from the pulmonary artery to the aorta, in which the blood-pressure will at this point be low.

In these two conditions the cause of the patency of the ductus arteriosus is readily explicable. Where there is no other developmental anomaly it is not so easy to understand. Imperfect expansion of the lungs during the earlier part of 
extra-uterine life might be invoked as an explanation, and the higher bloodpressure in the aorta than in the pulmonary artery would tend to perpetuate the condition.

It will be interesting to consider the effect that these anomalies may exercise on the mechanism of the circulation. In the normal condition (Fig. 5, $\alpha$ ), where the foetal passages are closed, it is clear that the same amount of blood will leave the left ventricle by the aorta as enters the right auricle by its venous orifices. In respect of the various functions of the four chambers of the heart we find the size of the cavity and the thickness of the wall of each to be that which we regard as normal.

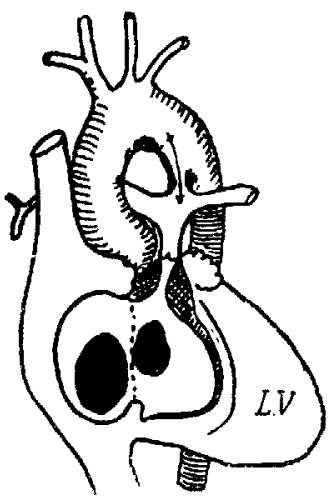

$\alpha$

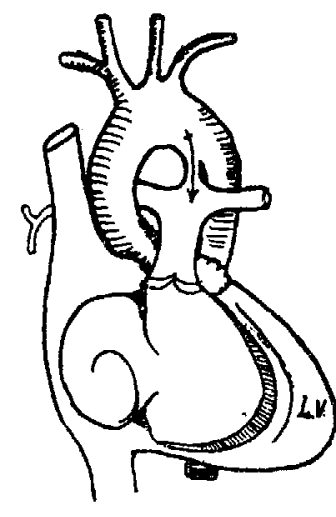

$b$

Fic. 6. (a) So-called pulmonary stenosis; (b) Simple patency of ductus arteriosus.

In coarctation of the aorta (Fig. 5, b) with a marked degree of constriction we should expect to find some diminution in the size and thickness of the left ventricle and of the first part of the aorta. The right side of the heart, on the other band, will have to deal with a larger amount of blood and will have the additional work imposed upon it of maintaining a considerable part of the systemic circulation, and so it will undergo dilatation and hypertrophy.

In arrest of development of the bulbus cordis, with 'pulmonary stenosis' (Fig. 6,a), there will usually be wide patency of the foramen ovale or of the interventricular aperture or of both. In consequence of this the right ventricle will dwindle in size and in thickness, and there will be dilatation and hypertrophy of the left ventricle, as it will have to accommodate a greater amount of blood and will have to maintain the greater part of the pulmonary as well as the systemic circulation.

When there is a patent ductus arteriosus without other anomaly (Fig. 6, $b$ ), and where, as is probable, the flow of blood is always from the aorta to the pulmonary artery, there will $I$ think be less blood circulating in that part of the system beginning in the aorta at the ope ing from this of the ductus, 
and ending at the opening of the inferior vena cava into the right auricle. In respect of this the right auricle will have less blood to deal with, and so consequently will the right ventricle and the trunk of the pulmonary artery. In consequence of the patency of the ductus arteriosus there will be an augmented flow of blood along the branches of the pulmonary artery, and this will prevail through the left side of the heart till we again reach the critical point where the ductus leads off from the aorta. In the outline diagrams I have made $I$ have tried to give expression to the changes in the size and thickness of wall of the various cavities of the heart under these various conditions.

I have said that there is not usually any cyanosis in cases of uncomplicated patency of the ductus arteriosus. Some of the blood will indeed pass twice through the lungs before going to the general system, a condition just the converse of that which prevails in cases of arrested development of the bulbus cordis with consequent patent ductus. On the other hand, the right ventricle will have some extra work imposed on it; though it may be dealing with a smaller amount of blood it has to drive that into a system in which the pressure is higher than normal. If it fails to do so satisfactorily there may result some stagnation of venous flow and some measure of cyanosis.

\section{CASE II.}

The second patient came under my observation when he was a boy of five years of age. He had never been strong, had always had some measure of bronchitis, and was suffering from some dyspnoea. There was some cyanosis and the veins of the face, neck, chest, and abdomen were all slightly prominent. The heart was enlarged. On July 27, 1906, I dictated the following note to the clinical clerk in charge of the case: "There is a systolic bruit which is louder in the aortic and pulmonary areas than at the apex. This is not louder in the pulmonary than in the aortic area. Behind it is high pitched and seems to last beyond the systole. In the episternal notch there is a thrill with its acme in the systole but not disappearing altogether during the diastole. There is no enlargement of the liver. The fingers and toes are slightly clubbed at their extremities.' As the clinical clerk had written that there was a systolic bruit audible with its maximum at the apex I can read into the above note an effort to correct his observation and perhaps some surprise that the bruit was not louder in the pulmonary area than in the aortic. I diagnosed congenital malformation of the heart, and stated that 'pulmonary stenosis' was the common condition found when there was cyanosis, but further than this I did not at that time go. The patient was again admitted under my care on April 18, 1914. There was slight cyanosis, but the lad was sharp and cheerful though rather undersized for his age, which was 13 . There was no enlargenent of the liver. The cardiac impulse was diffuse and was felt and seen one inch to the right of the sternum and one inch outside the left nipple line. It also extended further up than normal. The inner end of the left clavicle was seen to present a systolic elevation with each beat of the heart. There was wellmarked venous pulsation in the neck. No thrill could be made out anywhere. A diastolic thud was palpable to the left of the middle of the sternum. On auscultation the second sound was most marked at the inner end of the first 
interspace of the right side. At the apex the first sound was doubled. As the stethoscope was carried from the apex upwards and inwards, a faint bruit was found to lead off from the second sound when the left margin of the sternum was reached; this rapidly increased as the instrument was carried upwards and to the right, and when the inner end of the first right space was reached there was found to be a continuous humming sound with systolic and diastolic increments. These sounds were all heard at their maximum at this point, but they could be heard a considerable distance outwards along the first right interspace. At the back of the chest the continuous hum could be heard on both sides of the spine, but it was louder on the right side than on the left. It could be heard as far down as the sixth dorsal spine, but its maximum was at the fourth. The bruit I have mentioned had very much the same characters as that in the case $I$ have already detailed. It was quite continuous and conformed in all respects except in situation to Gibson's classical description.
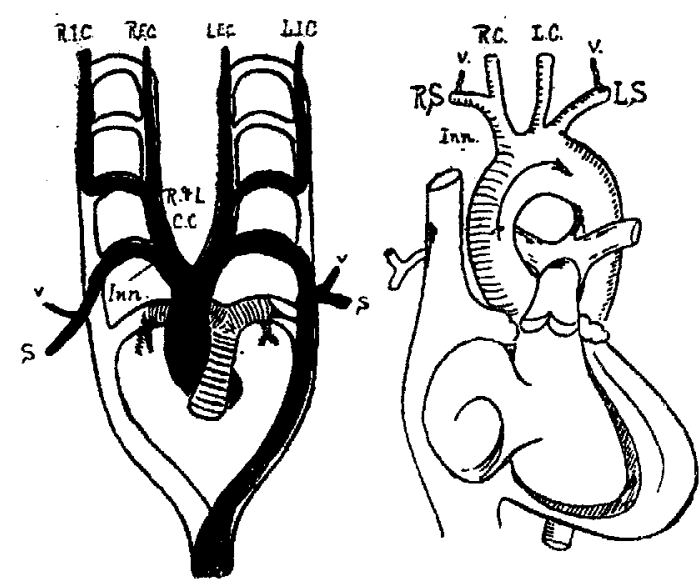

FIG. 7. To show the development of the normal condition with the left aortic arch. (In the schema of this figure the permanent systemic vessels are shown by the thick black shading, the pulmonary vessels by cross marking, and the fibrous remains of the ductus arteriosus by a thin black line.)

It did not appear to me that any valvular lesion could account for the bruit met with in this case. There appeared to be two conditions only which could account for the sounds heard, and these were some communication between the aorta and the superior vena cava or a patent ductus arteriosus. A continuous humming sound, with systolic and, it might be, diastolic exacerbations, might well be heard in this situation if there were an aperture of communication between the aorta and the superior vena cava. In a young lad it would, on the one hand, be hard to suggest a cause for such a condition, and, on the other hand, if such a communication did exist we should expect marked distension and tortuosity of the veins of the neck and chest, for the thin-walled superior vena cava and its tributaries are ill adapted to resist the pressure generated by the contraction of the left ventricle.

If the alternative view of patency of the ductus arteriosus is entertained, we are met with the difficulty that the signs are on the right side and not on the left. The normal development of the great vessels is shown in Fig. 7, where it is seen that the ductus arteriosus is derived from the distal part of the fifth 


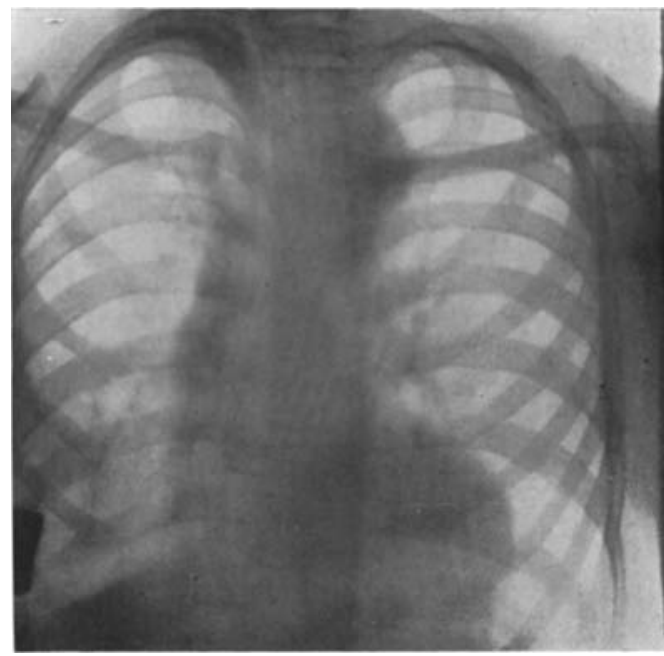

FIG. 9. Shows the extension of the supracardiac shadow to the right, and the well-defined extension upwards and to the left under the clavicle. 
left branchial arch. A condition of complete transposition of the viscera would account for the alonormal area in which the sound was heard, but the apex beat was palpable to the left of the sternum and the liver dullness was on the right side, so wo had to exclude situs inversus.

Sometimes, however, transposition of the arch of the aorta without transposition of the heart is met with, and most anatomical museums are able to show one or more specimens of this anomaly. The development of this condition is shown in Fig. 8. The first part of the aorta has its usual relation to the pulmonary artery, being at first behind it and then to the right-hand side. From this the aorta arches over the root of the right lung instead of the left, and passes to the back of the chest on the right of the spine. When this is the case the ductus arteriosus may be developed on one side or on the other. If it is
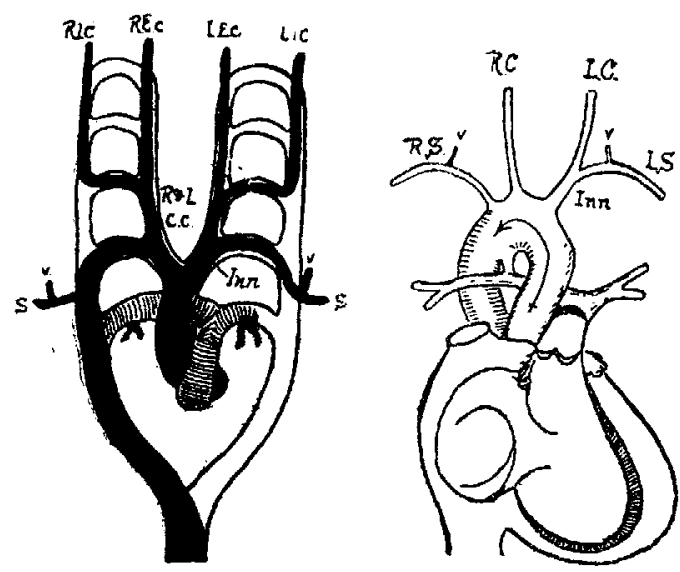

FIG. 8. To show the development of a right aortic arch without transposition of the heart, ductus (right-sided) being patent.

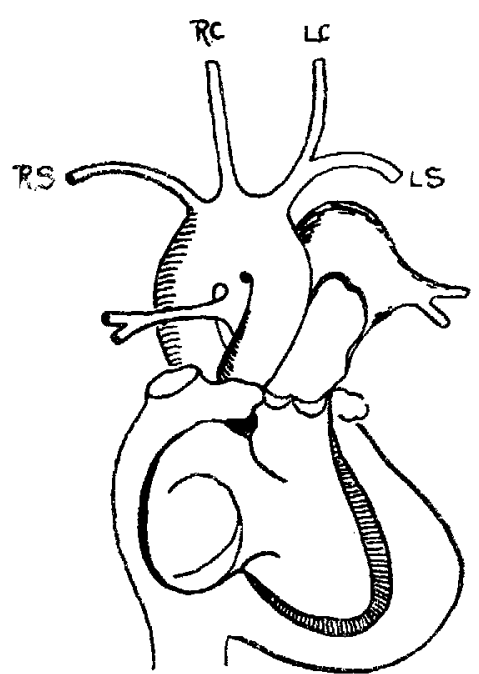

Fia. 10. To show the condition believed to be present; a right arch, without transposition of the heart; ductus arteriosus developed on the right side and remaining patent; greatly dilated pulmonary axtery.

developed from the distal part of the fifth right branchial arch, the humming bruit with its systolic and diastolic exacerbations would be heard as it was in the case under discussion, on the right side rather than on the left.

Dr. Rowden made an examination with the radiographic screen and came to the conelusion that there was a distinct widening of the supracardiac area of opacity referable to the great vessels. This extended further to the right than usual and the appearance of its right margin was suggestive of an aortic origin. A well-marked extension of the opaque area passed upwards and to the left, and presented very distinct pulsation. It was clearly this which caused the systolic elevation of the inner end of the clavicle. The skiagram (Fig. 9) shows some of these points, but not so well as the fluoroscopic screen.

After a full consideration of the findings by the various means of investi- 
gation I incline to think the condition may be that which I have represented in Fig. 10, in which there is transposition of the arch of the aorta without transposition of the heart, and in which the ductus arteriosus has been developed, and has remained patent, on right side. It is assumed that there is great dilatation of the pulmonary artery and that this is accountable for the pulsating shadow extending up towards the left clavicle.

It may be that the cyanosis has its explanation in the difficulty which the right ventricle experiences in discharging its blood into the pulmonary artery, seeing that this is being fed from the aorta by the patent ductus. On the other hand, there may be some further cardiac anomaly. I have, however, seen too many cases of the morbus coeruleus, and made too many autopsies for the investigation of the underlying conditions, to venture on any definite suggestions. as to what that further anomaly may be.

\section{REFERENCES.}

1. Edinb. Med. Jourm., 1900, N. S., viii. 212.

2. Lancet, Lond., 1906, ii. 973.

3. Amer. Journ. Med. Sci., 1913, N. S., exlv. 543.

4. Allbutt and Rolleston, System of Medicine, 2nd ed., 1909, vi. 310.

5. Edinb. Med. Journ., 1884, xxx. 17.

6. 'Malformations of the Bulbus Cordis', Aberdeen University Quatercentenary Publications. 